\title{
The Tobacco mosaic virus Replicase Protein Disrupts the Localization and Function of Interacting Aux/IAA Proteins
}

\author{
Meenu S. Padmanabhan, ${ }^{1}$ Haiymanot Shiferaw, ${ }^{2}$ and James N. Culver ${ }^{1,2}$ \\ ${ }^{1}$ Department of Cell Biology and Molecular Genetics, University of Maryland, and ${ }^{2}$ Center for Biosystems Research, \\ University of Maryland Biotechnology Institute, College Park, MD 20742, U.S.A.
}

Submitted 24 February 2006. Accepted 26 March 2006.

Previously, we identified a correlation between the interaction of the Tobacco mosaic virus (TMV) 126/183-kDa replicase with the auxin response regulator indole acetic acid (IAA)26/PAP1 and the development of disease symptoms. In this study, the TMV replicase protein is shown to colocalize with IAA26 in the cytoplasm and prevent its accumulation within the nucleus. Furthermore, two additional auxin (Aux)/IAA family members, IAA27 and IAA18, were found to interact with the TMV replicase and displayed alterations in their cellular localization or accumulation that corresponded with their ability to interact with the TMV replicase. In contrast, the localization and accumulation of noninteracting Aux/IAA proteins were unaffected by the presence of the viral replicase. To investigate the effects of the replicase interaction on Aux/IAA function, transgenic plants expressing a proteolysis-resistant IAA26-P108Hgreen fluorescent protein (GFP) protein were created. Transgenic plants accumulating IAA26-P108H-GFP displayed an abnormal developmental phenotype that included severe stunting and leaf epinasty. However, TMV infection blocked the nuclear localization of IAA26-P108H-GFP and attenuated the developmental phenotype displayed by the transgenic plants. Combined, these findings suggest that TMV-induced disease symptoms can be attributed, in part, to the ability of the viral replicase protein to disrupt the localization and subsequent function of interacting Aux/IAA proteins.

Additional keywords: subcellular localization, virus disease.

Plant virus infections often disturb host physiology and result in the display of disease symptoms. However, disease severity does not typically correlate with the ability of a virus to replicate or accumulate to high levels, indicating that the diversion of cellular metabolites toward virus synthesis is not a primary factor in the disease process (Hull 2002). In addition, the induction of specific disease symptoms is often heritable and characteristic for certain virus-host combinations, suggesting that interactions between specific virus and host components are key factors in disease development. Although genetic changes in both viral and host components have been correlated with altered disease phenotypes, the mechanisms through which these components function to control disease are not well characterized. A better understanding of these

Corresponding author: J. N. Culver: E-mail: jculver@umd.edu; Fax: +1. 301. 314.9075. mechanisms represents an important step in the development of new strategies for reducing the economic impact of plant virus diseases.

Tobacco mosaic virus (TMV) is the type member of the genus Tobamovirus and functions as a model for the study of virushost interactions. The TMV 126-kDa and read-through 183$\mathrm{kDa}$ replicase proteins have been linked to the induction of various disease symptoms (Bao et al. 1996; Shintaku et al. 1996). Biochemical characterizations of the 126-kDa protein have demonstrated guanylyltransferase-like activity that results in the capping of viral RNAs as well as NTPase and RNAunwinding activity derived from the $\mathrm{C}$-terminal helicase domain (Dunigan and Zaitlin 1990; Erickson et al. 1999; Goregaoker and Culver 2003; Merits et al. 1999). Specificity for 3' RNA binding has been mapped to a 110-amino-acid region downstream of the methyl transferase domain (Osman and Buck 2003). Tobamovirus replicase proteins also play a role in virus cell-to-cell movement (Goregaoker et al. 2001; Hirashima and Wantanabe 2001; Liu et al. 2005) and function in the suppression of gene silencing (Ding et al. 2004; Kubota et al. 2003). Interestingly, Tobamovirus replicase proteins are implicated in several host interactions that affect virus replication and symptom development. These interactions include association with the RNA binding subunit of eIF-3 (Osman and Buck 1997), replication-dependent association with host membrane proteins (Yamanaka et al. 2000), elicitation of $\mathrm{N}$ gene-mediated disease resistance (Abbink et al. 2001; Erickson et al. 1999; Padgett and Beachy 1993), a host cell death response modulated by a plant P58IPK-like inhibitor of double-stranded RNA-activated protein kinase (Bilgin et al. 2003), and association with a plant ATPase and a component of the photosystem II oxygen-evolving complex that affects virus accumulation (Abbink et al. 2002). Combined, these data indicate that the Tobamovirus replicase plays a multifunctional role in the infection process.

Interestingly, most Tobamovirus replicase interactions have been mapped to the helicase domain, suggesting an importance for this motif in viral pathogenesis. Previously, we described an interaction between the auxin/indole acetic acid (Aux/IAA) protein IAA26/PAP1 and the helicase domain of the TMV replicase protein (Padmanabhan et al. 2005). IAA26 belongs to a 29-member family of Aux/IAA transcription factors (Liscum and Reed 2002). Members of this family encode short-lived nuclear proteins that mediate auxin-dependent gene expression (Abel et al. 1994; Ulmasov et al. 1997b). Sequence analysis has shown that almost all Aux/IAA proteins have four conserved motifs (domain I, II, III, and IV) (Abel et al. 1994). Domain II mediates the rapid auxin-dependent turnover of these proteins (Ramos et al. 2001; Zenser et al. 2003). Domains III and IV, 
located in the $\mathrm{C}$ terminal end, mediate protein-protein interacttions between Aux/IAA proteins and auxin-response factor proteins (ARFs), which bind auxin-response elements (AuxREs) in the promoters of auxin-responsive genes (Kim et al. 1997; Ulmasov et al. 1995, 1997a). The current model for auxin signaling suggests that, at low auxin concentrations, Aux/IAA proteins modulate the activity of interacting ARFs (Leyser 2002; Reed 2001; Ulmasov et al. 1997a). At higher auxin concentrations, Aux/IAA proteins are targeted for degradation by the $\mathrm{SCF}^{\mathrm{TIR} 1}$ ubiquitin ligase complex, allowing ARFs to function as either activators or repressors of AuxRE-containing genes (Dharmasiri and Estelle 2004; Gray et al. 2001). Auxincontrolled proteolysis of Aux/IAA proteins provides a sensitive method for the control of genes involved in numerous developmental processes, including cell expansion and differentiation, tropic responses to light and gravity, organ patterning, vascular development, and apical dominance.

Molecular characterization of the IAA26-TMV replicase interaction suggested that IAA26 function was disrupted in infected tissue resulting in the display of disease symptoms (Padmanabhan et al. 2005). In this study, we demonstrate that the TMV replicase directly inhibits the nuclear localization of IAA26. Furthermore, two additional Aux/IAA family members, IAA27/PAP2 and IAA18, also interact with the TMV replicase protein and show changes in localization that correspond to the strength of the replicase interaction. In contrast, the localization of noninteracting Aux/IAA proteins was not affected by the presence of the TMV replicase. TMV infection also reversed stunting and epinasty associated with the abnormal developmental phenotype of transgenic plants that accumulated a proteolysis-resistant mutant of IAA26. Thus, interference in the nuclear localization of Aux/IAA proteins provides a mechanism for the disruption of auxin-mediated plant development and the induction of disease.

\section{RESULTS}

\section{The TMV 126-kDa replicase protein disrupts IAA26 nuclear localization.}

Previously, we demonstrated that interaction between the TMV replicase and IAA26 corresponded with altered accumulations of IAA26 in infected tissues (Padmanabhan et al. 2005). To determine the direct effects of the replicase interaction on IAA26 localization, two transient expression constructs, IAA26-DsRed (Discosoma sp. Red fluorescent protein) and TMV126-green fluorescent protein (GFP), were created and coexpressed in onion epidermal cells. When expressed alone, IAA26-DsRed localized almost exclusively to the nucleus and TMV126-GFP localized to the cytoplasm-forming vesicle-like bodies (Fig. 1A to D). These vesicle-like bodies are derived from the endoplasmic reticulum (ER) and are consistent with membrane-associated virus replication complexes (VRC) formed during virus replication (Figueira et al. 2002; Liu et al. 2005; Más and Beachy 1999). When the two fusion constructs were coexpressed, IAA26-DsRed displayed a dramatic shift in localization from the nucleus to the cytoplasm and colocalized almost exclusively with the vesicle-like bodies produced by TMV126-GFP (Fig. 1E to H). To examine replicase specificity in the observed alteration in IAA26 localization, a TMV 126$\mathrm{kDa}$ construct, TMV126 $\mathrm{HEL}-\mathrm{GFP}$, containing a deletion of the helicase domain of amino acids 782 to 1,116 , also was analyzed in the colocalization assay. Although TMV126 $\Delta$ HELGFP retained a localization pattern similar to the full-length TMV126-GFP, it did not significantly alter the nuclear localization of IAA26-DsRed (Fig. 2). Thus, interference in the nuclear localization of IAA26 is dependent upon the presence of the viral helicase domain.
Additional Aux/IAA members interact with the TMV helicase domain.

Because IAA26 belongs to a large family of related proteins, the possibility existed that other Aux/IAA members also interacted with the viral replicase. To test this possibility, additional Aux/IAA family members sharing varying degrees of homologies with IAA26 were investigated for their ability to interact with the TMV helicase domain (Fig. 3). Within the two-hybrid system, none of the nine tested Aux/IAA members strongly interacted with the TMV helicase domain at a level similar to that observed for IAA26. However, two Aux/IAA members, IAA27 and IAA18, displayed reduced yet significant interaction levels that were approximately 47 and $13 \%$ that of IAA26, respectively (Fig. 3).

\section{Interaction with the TMV replicase affects the localization of related Aux/IAA members.}

To investigate the ability of the TMV replicase to directly affect the localization of other Aux/IAA family members, the open reading frames (ORFs) of IAA27, IAA18, IAA12, and IAA2 were fused to DsRed and coexpressed with TMV126GFP in onion epidermal cells. These Aux/IAA members possess a range of abilities to interact with the TMV helicase domain (Fig. 3). In the presence of TMV126-GFP, IAA27-DsRed showed a noticeable change in localization when compared with similar experiments done in the absence of TMV126-GFP (Fig. 4A and E). Most significantly, IAA27-DsRed produced only weak accumulations of DsRed fluorescence in the nucleus and the appearance of aggregates that colocalized with TMV126-GFP. In contrast, IAA18-DsRed, IAA12-DsRed, and IAA2-DsRed did not display a noticeable change in localization when coexpressed with TMV126-GFP (Fig. 4B and F, C and $\mathrm{G}$, and $\mathrm{D}$ and $\mathrm{H}$ ).

To further address the biological relevance of the colocalization studies, GFP fusion constructs also were created for IAA27, IAA18, IAA12, and IAA2 and transiently expressed in either mock-inoculated or TMV-infected leaf tissue (Fig. 4). All four Aux/IAA-GFP fusion constructs localized tightly to the nucleus in mock-inoculated tissues. However, in TMVinfected tissue, IAA27-GFP fluorescence appeared predominately as cytoplasmic inclusions (Fig. 4I and M). In addition, the total number of cells displaying IAA27-GFP fluorescent in TMV-infected tissues was only $35 \%$ of the total observed in mock-inoculated tissues (Fig. 5). For IAA18-GFP, fluorescence resided primarily in the nucleus; however, the total number of cells displaying IAA18-GFP fluorescence was just $50 \%$ of that observed in mock-inoculated tissues (Figs. 4J and $\mathrm{N}$, and 5). For nonhelicase-interacting IAA members IAA12-GFP and IAA2-GFP, fluorescence appeared exclusively in the nucleus in both mock- and TMV-infected tissues (Fig. 4K and $\mathrm{O}$, and $\mathrm{L}$ and $\mathrm{P}$ ). Furthermore, the number of cells displaying IAA12-GFP fluorescence was not significantly different between mock- and TMV-infected tissues (Fig. 5). Collectively, these findings indicate a correlation between the strength of the replicase-Aux/IAA interaction and the ability of the TMV replicase to disrupt Aux/IAA localization.

\section{Construction of an auxin-resistant IAA26.}

To address the affects of the TMV replicase-Aux/IAA interaction in plants, a 35S:IAA26-GFP construct was used to transform Arabidopsis thaliana ecotype Shahdara. This ecotype was selected because previous studies had shown that TMV spreads and accumulates at levels that are comparable with other hosts, such as tobacco and tomato (Dardick et al. 2000). We assumed that overexpression of IAA26 would result in developmental abnormalities that could be used to function- 
ally assess the IAA26-replicase interaction during virus replication. However, transformed plants confirmed by reversetranscriptase polymerase chain reaction (RT-PCR) for the accumulation of the transgene mRNA showed no detectable levels of the IAA26-GFP protein or GFP fluorescence (data not shown). These plants also displayed no significant change in phenotype when compared with nontransformed plants. Similar results have been reported for the overexpression of other wild-type Aux/IAAs (Gray et al. 2001; Park et al. 2002; Rogg et al. 2001). Park and associates (2002) speculated that, due to their extremely short half-lives, these proteins are unable to accumulate when overexpressed.

To overcome the rapid turnover associated with wild-type Aux/IAA proteins, a $\mathrm{P}$ to $\mathrm{L}$ substitution was introduced at residue 108 in domain II of IAA26. Similar mutations in other Aux/IAA members have resulted in the enhanced stability and accumulation of the mutant protein even in the presence of excess auxin (Gray et al. 2001; Kepiski and Leyser 2002; Ouellet et al. 2001; Tian et al. 2003). Stability of the IAA26-P108H-
GFP constructs was tested in transient expression assays using onion epidermal cells. Results indicated that, in the presence of excess IAA (1 mM), IAA26-P108H-GFP remained stable within the nucleus while the wild-type IAA26 degraded rapidly (Fig. 6A and B). Clearly, the addition of the P108L mutation significantly enhanced auxin resistance and the stability of IAA26.

Two hybrid interaction studies demonstrated that the IAA26-P108H mutation did not significantly affect the interaction of IAA26 with the TMV helicase domain (Fig. 6C). In addition, IAA26-P108H-GFP showed markedly different localization patterns in mock- and TMV-infected tissues. In mockinoculated tissues, IAA26-P108H-GFP showed only nuclear localization. In TMV-infected tissues, IAA26-P108H-GFP was localized predominately within the cytoplasm in small vesiclelike inclusions (Fig. 6D). These findings are consistent with results obtained using the unmodified IAA26 protein and indicate that this mutation does not significantly affect the TMV replicase-IAA26 interaction (Padmanabhan et al. 2005).
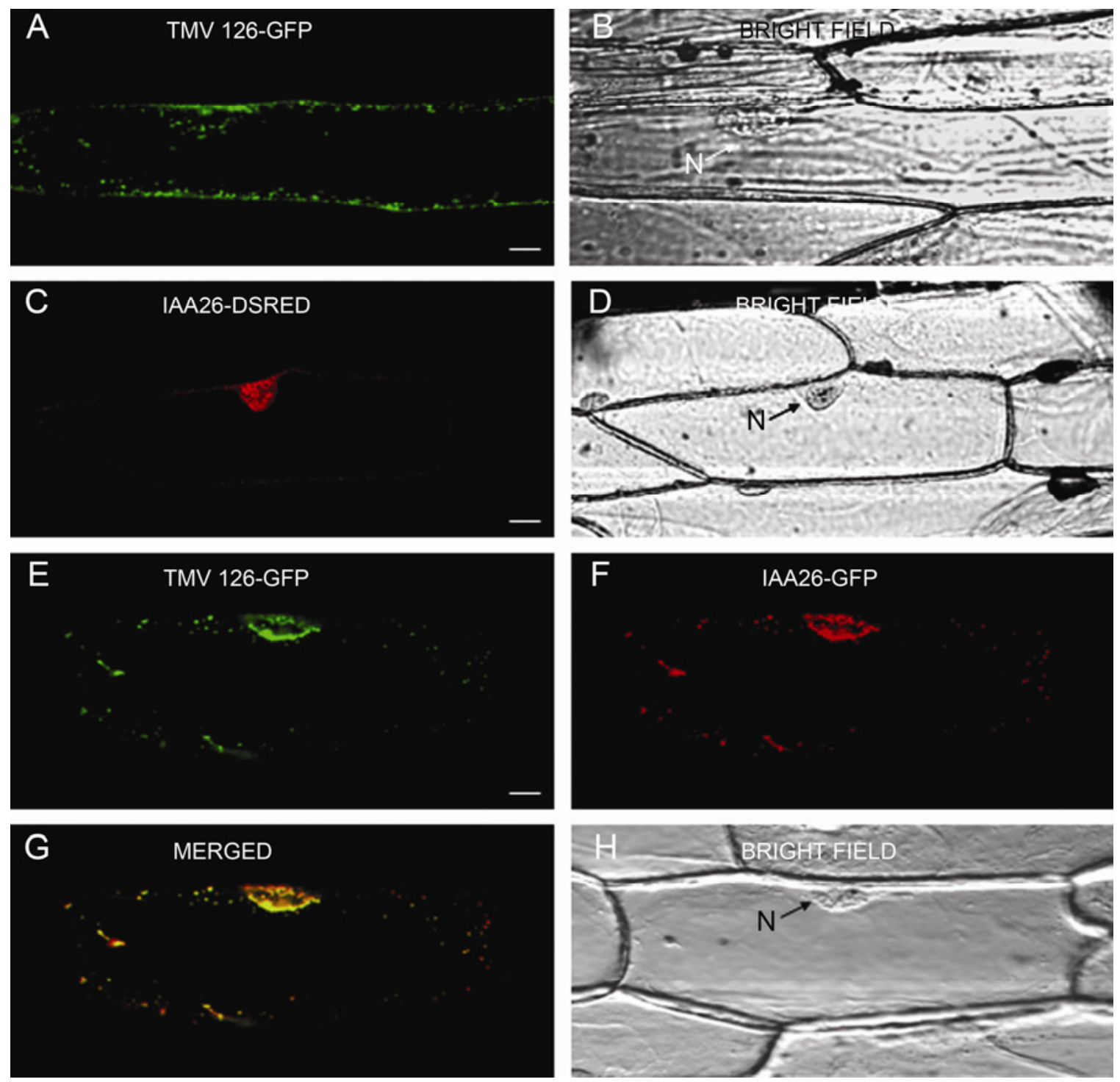

Fig. 1. Nuclear localization of indole acetic acid (IAA)26 is altered in the presence of the Tobacco mosaic virus (TMV) 126-kDa replicase protein. Fusion proteins were transiently expressed in onion epidermal cells via particle bombardment and fluorescent images captured $20 \mathrm{~h}$ post-transformation. For coexpression studies, equal concentrations of expression plasmids were mixed and bombarded together. Nucleus is labeled N. Bar $=20 \mu \mathrm{m}$. A and $\mathbf{B}$, Fluorescence and bright-field images showing the localization pattern of TMV 126-kDa replicase protein fused to green fluorescent protein (GFP). C and D, Fluorescence and bright-field images of cells showing nuclear localization of IAA26 fused to Discosoma sp. Red fluorescent protein (DsRed). E, F, G, and $\mathbf{H}$, Coexpression of IAA26-DsRed and TMV126-GFP. E, TMV126-GFP fluorescence. F, IAA26-DsRed fluorescence. G, Overlay of fluorescence from $\mathbf{E}$ and $\mathbf{F}$. H, Bright-field image of the coexpressing cell. 
Interaction with the TMV replicase affects IAA26 function. A. thaliana ecotype Shahdara transformed with 35S:IAA26-P108H-GFP was generated to test the affects of TMV infection on the function of IAA26. In contrast to the wild-type IAA26-GFP transformed plants, fluorescent microscopy revealed the presence of nuclear-localized GFP in young seedlings (Fig. 7B). In mature leaf tissues used for virus inoculations, GFP fluorescence was observed only occasionally; however, the presence of the IAA26-P108H-GFP protein was readily detected in these tissues by Western immunoblot analysis (Fig. 7C). Similar age-dependent differences in GFP fluorescence previously have been accredited to higher levels of chlorophyll interference in older tissue (Zhou et al. 2005). Plants accumulating IAA26P108H-GFP displayed a severely stunted phenotype along with leaf epinasty (Fig. 7A). This phenotype is consistent with that observed in other studies that have utilized auxinresistant Aux/IAA mutants (Gray et al. 2001; Kepiski and Leyser 2002; Tian et al. 2003). However, when inoculated with TMV, IAA26-P108H-GFP plants showed a marked attenuation in the severity of this phenoltype. Most notably, the severe stunting and epinasty in these plants decreased over a period of 1 to 2 weeks postinoculation and was more pronounced in tissues that developed after inoculation (Fig. 7D). Decreases in the severity of the IAA26-P108H-GFP phenotype corresponded with the virus's ability to spread and accumulate to high levels within newly developing tissues (Dardick et al. 2000).
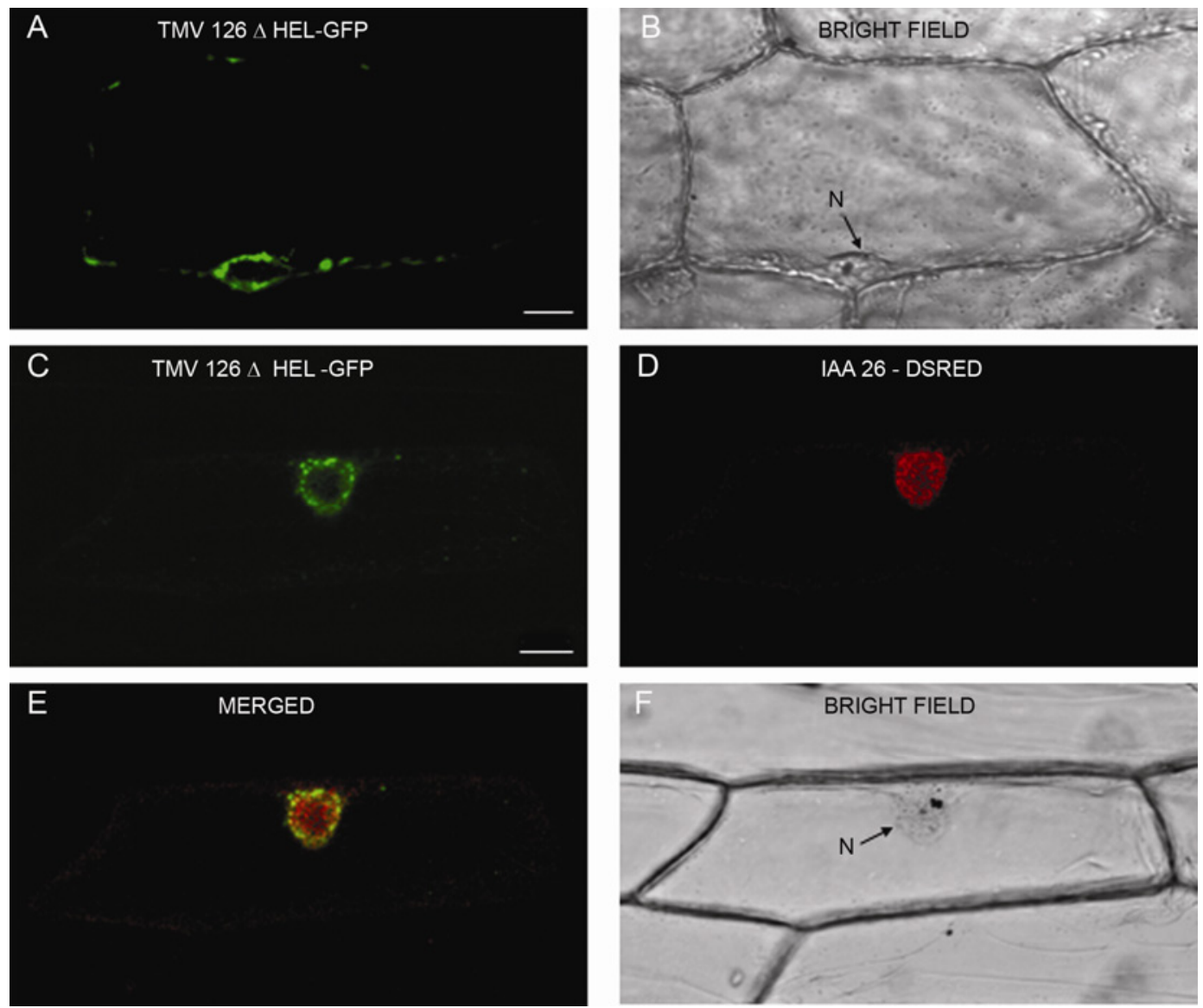

Fig. 2. Deletion of the helicase domain negates the effect of replicase on indole acetic acid (IAA)26 localization. Tobacco mosaic virus (TMV)126 $\triangle$ HEL-green fluorescent protein (GFP) contains a deletion of amino acids 782 to 1,116 required for the replicase-IAA26 interaction. N indicates the location of the nucleus. Images were visualized $20 \mathrm{~h}$ post-transformation. Bars $=20 \mu \mathrm{m}$. A and B, Fluorescence and bright-field confocal images of an onion epidermal cell transiently expressing TMV126- $\Delta$ HEL-GFP fusion protein. C, D, E, and F, Co-expression of TMV126- $\Delta$ HEL-GFP and IAA26DsRed fusion proteins in onion epidermal cells. C, TMV126- $\Delta$ HEL-GFP- and D, IAA26-DsRed-derived fluorescence. E, Overlay of fluorescence images $\mathbf{C}$ and $\mathbf{D}$. F, Bright-field image of the cell. 
and Beachy 1999; Szecsi et al. 1999). Previous studies have shown that the TMV 126-kDa replicase protein associates with the ER (primarily the peri-nuclear ER), promotes alterations in its morphology, and is sufficient to induce the formation of vesicle-like structures (Beachy and Zaitlin 1975; Figueira et al. 2002; Liu et al. 2005; Mathews 1981; Más and Beachy 2000; Reichel and Beachy 1998; Shalla 1964). Interestingly, colocalization studies with the TMV $126-\mathrm{kDa}$ replicase protein and interacting IAA26 revealed that the interaction between the two proteins is sufficient to alter the subcellular localization of IAA26 such that it accumulates around the peri-nuclear ER and within the vesicle-like inclusions produced by the 126 $\mathrm{kDa}$ protein. Deletion of the interacting helicase domain or use of a noninteracting Aux/IAA protein did not result in colocalization, indicating that the effect of replicase on Aux/IAA localization is dependent on the ability of these proteins to associate. Thus, sequestration of interacting Aux/IAAs within vesicle-like replicase complexes provides a mechanism whereby the virus can directly interfere in the auxin-regulated development of the plant. Combined with previous findings that linked this interaction to the display of disease symptoms (Padmanabhan et al. 2005), it is likely that TMVinduced disease symptoms are derived at least partially from the ability of the viral replicase to disrupt the localization of interacting Aux/IAA proteins.
Aux/IAA proteins make up a conserved family of auxinresponsive transcription factors engaged in the regulation of plant development (Liscum and Reed 2001). Conserved similarities between Aux/IAA family members suggested that the

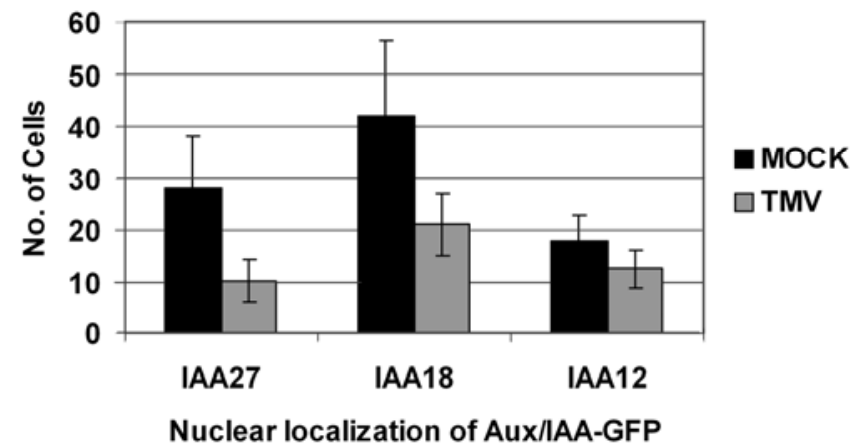

Fig. 5. Expression and nuclear localization of indole acetic acid (IAA)27green fluorescent protein (GFP), IAA18-GFP, and IAA12-GFP constructs in noninfected (Mock) and Tobacco mosaic virus (TMV)-infected Nicotiana benthamiana leaf tissues. Values are the mean number of cells \pm standard deviations (error bars) within a $15-\mathrm{mm}^{2}$ leaf area visualized $16 \mathrm{~h}$ posttransformation and averaged from five independent bombardment transformations. Aux = auxin.
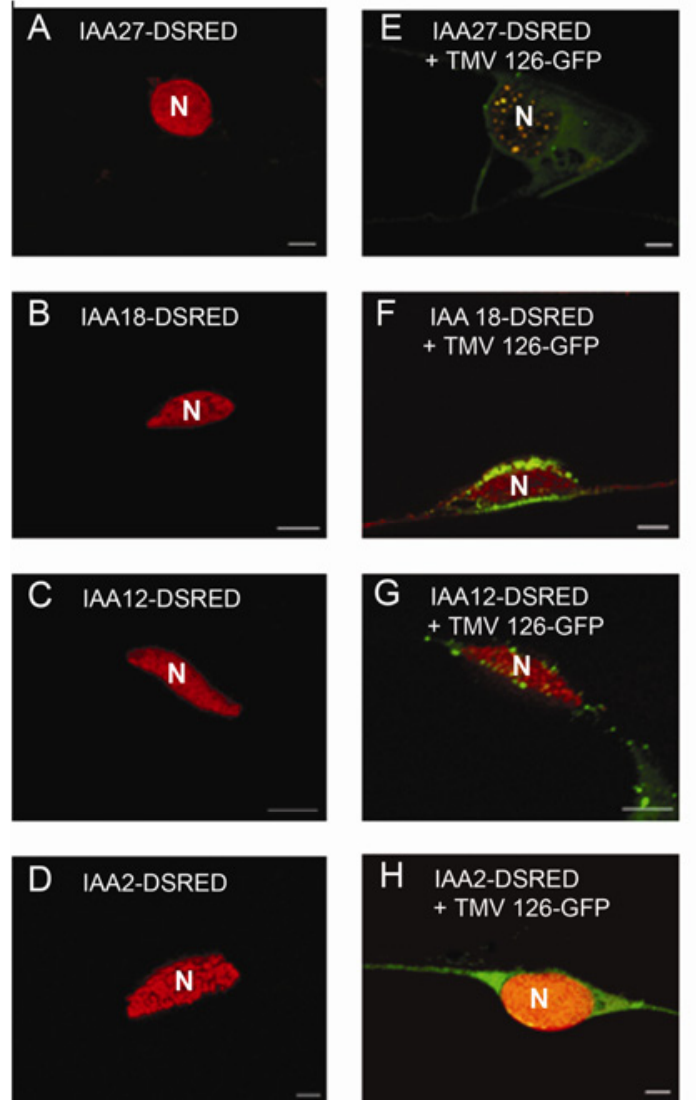

MOCK INFECTED
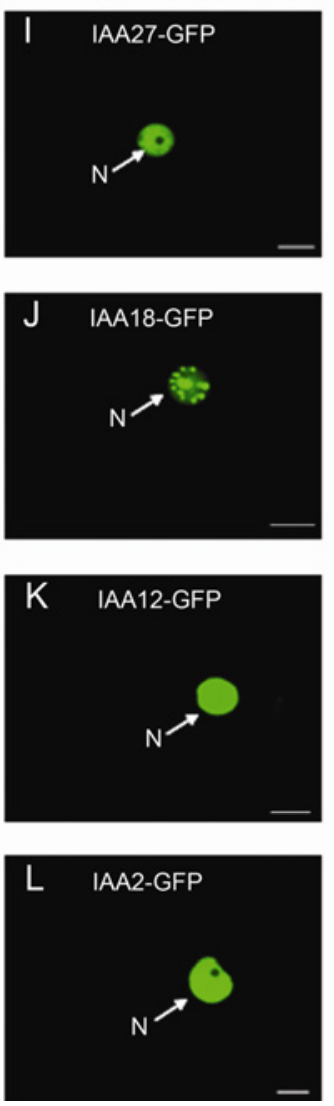
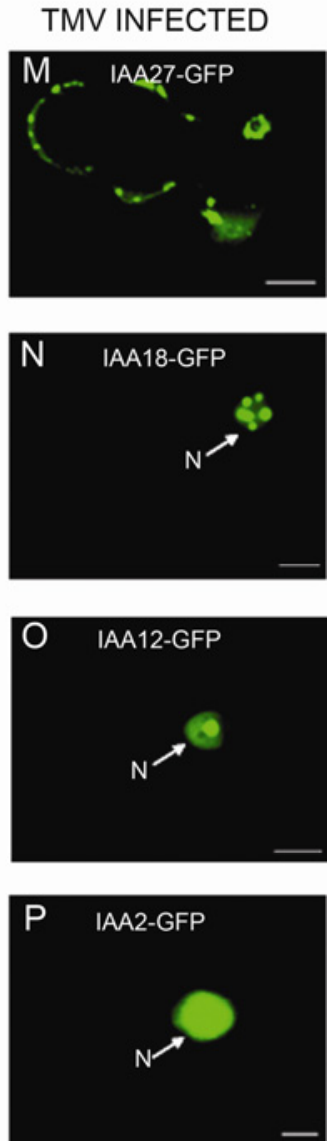

Fig. 4. Tobacco mosaic virus (TMV) replicase interacts with and alters the nuclear localization of indole acetic acid (IAA) 27 but not IAA18, IAA12, or IAA2. Fusion protein expression was visualized $20 \mathrm{~h}$ post-transformation in onion epidermal cells and $16 \mathrm{~h}$ post-transformation in Nicotiana benthamiana leaf tissue. The nucleus is labeled N. Bars $=10 \mu \mathrm{m}$. A, B, C, and $\mathbf{D}$, Confocal images of auxin (Aux)/IAA proteins fused to Discosoma sp. Red fluorescent protein (DsRed) and transiently expressed in onion epidermal cells. A, IAA27-DsRed. B, IAA18-DsRed. C, IAA12-DsRed. D, IAA2-DsRed. E, F, G, and H, Confocal images of onion epidermal cells co-expressing Aux/IAA-DsRed proteins with TMV126-green fluorescent protein (GFP). E, IAA27-DsRed. F, IAA18-DsRed. G, IAA12-DsRed. H, IAA2-DsRed. I, J, K, and $\mathbf{L}$, Confocal images of Aux/IAA proteins fused to GFP and transiently expressed in N. benthamiana leaf tissue mock inoculated with distilled water. I, IAA27-GFP. J, IAA18-GFP. K, IAA12-GFP. L, IAA2-GFP. M, N, O, and P, Confocal images of Aux/IAA proteins fused to GFP and transiently expressed in $N$. benthamiana leaf tissue infected with TMV. M, IAA27-GFP. N, IAA18-GFP. O, IAA12GFP. P, IAA2-GFP. 
TMV replicase could affect the localization of other Aux/IAA proteins. A two-hybrid screen using Aux/IAA proteins with a range of sequence similarities to IAA26 identified IAA27 and IAA18 as interacting with the TMV helicase domain, albeit at a reduced levels. Localization studies revealed a correlation between the strength of the Aux/IAA-replicase interaction and the level of interference in Aux/IAA localization. Most significantly, the ability of Aux/IAA-GFP constructs to localize to the nucleus in TMV-infected tissues versus mock-inoculated tissues was less than 5\% for IAA26 (Padmanabhan et al. 2005), approximately 35 and 50\% for IAA27 and IAA18, respectively. For noninteracting Aux/IAAs, such as IAA12, there was no significant difference in nuclear localization in infected versus mock-inoculated tissues. Colocalization studies also supported this correlation, with only the two strongest interactors, IAA26 and IAA27, displaying significant localization changes in the presence of the TMV replicase. The weakly interacting IAA18 appears to be near the threshold needed for the replicase to affect its localization. It was not affected in transient expression assays and only partially altered in infected tissues where replicase accumulations would be greater. Interestingly, there was no direct correlation between Aux/IAA sequence similarity and the strength of the TMV replicase interaction. For example, IAA18 shares the greatest sequence

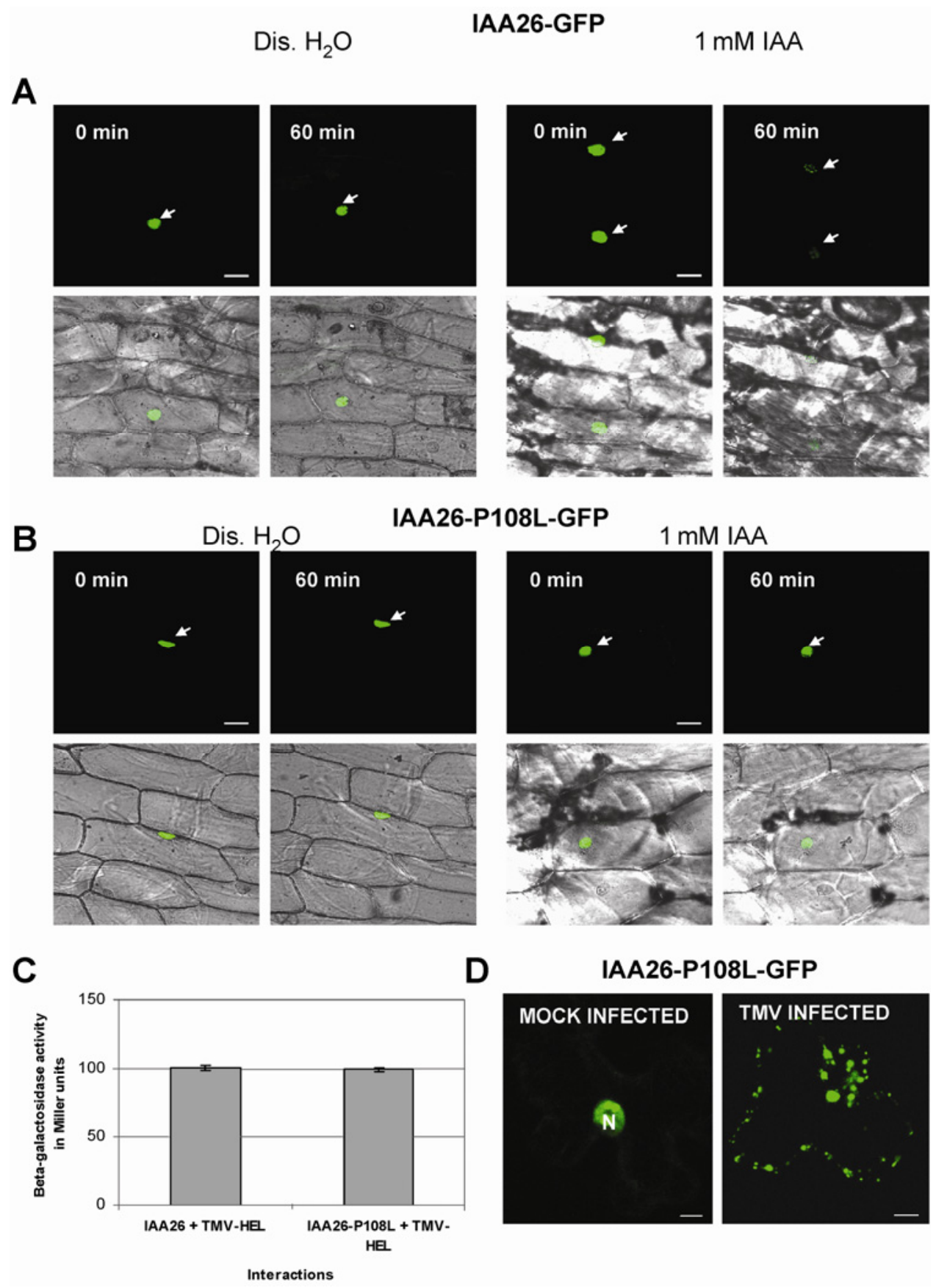

Fig. 6. Indole acetic acid (IAA)26-P108L-green fluorescent protein (GFP) is resistant to auxin-mediated degradation but retains its ability to interact with the Tobacco mosaic virus (TMV) replicase. A, IAA26-GFP and B, IAA26-P108H-GFP. Photos represent fluorescence and light microscopy images of onion epidermal cells expressing the two GFP fusion proteins. Onion cells were treated with water or $1 \mathrm{mM}$ IAA and monitored for $1 \mathrm{~h}$. Bars $=50 \mu \mathrm{m}$. Arrows indicate the nucleus. C, Quantitative $\beta$-galactosidase activity for yeast-two-hybrid interactions between the TMV helicase and IAA26 or IAA26-P108H. D, Transient expression and visualization of IAA26-P108H-GFP in mock-infected or TMV-infected Nicotiana benthamiana leaf tissue. Images obtained $16 \mathrm{~h}$ post-transformation. $\mathrm{N}$ indicates nucleus. Bar $=10 \mu \mathrm{m}$. 
similarity to IAA26, yet it interacts only weakly with the TMV helicase domain, whereas the less-similar IAA27 interacts more strongly (Fig. 3). Lack of a correlation between sequence similarity and replicase interaction suggests that the TMV helicase domain interacts with Aux/IAA structural features that are not readily identifiable from the primary sequence. Thus, other Aux/IAA members not tested in this study cannot be excluded as potential targets for replicase interaction. The ability of the TMV replicase protein to alter the localization of multiple Aux/IAA proteins expands the potential impact of the virus on the plant's auxin-sensing pathway and the development of disease.

The ability of the TMV replicase protein to alter the localization of Aux/IAA proteins in vivo suggests that this interacttion could significantly disrupt the normal transcriptional control mediated by interacting Aux/IAA proteins. This possibility is supported by the finding that approximately $30 \%$ of the genes that display transcriptional alterations in response to
TMV infection contain two or more AuxREs within their promoters (Padmanabhan et al. 2005). To determine whether TMV infection can disrupt the function of an interacting Aux/IAA protein, a point mutation in domain II of IAA26 (IAA26-P108H) was created to enhance its resistance to proteolysis and to induce a defined developmental phenotype when overexpressed as a transgene. A large body of work had shown that, in many Aux/IAA proteins, domain II and its conserved proline residue form a key structural module that is necessary for auxin-mediated interaction with the E3 ubiquitin ligase complex-SCF ${ }^{\mathrm{TIR} 1}$, ultimately leading to the degradation of the protein via the $26 \mathrm{~S}$ proteasome (Gray et al. 2001; Kepinski and Leyser 2004, 2005). Several gain-of-function Aux/IAA mutants have a substitution in the conserved proline residue and all these mutants show enhanced stability of the protein in the presence of auxin and a diverse set of auxin-responserelated phenotypes (Leyser et al. 1996; Nagpal et al. 2000; Reed 2001; Rogg et al. 2001; Rouse et al. 1998). When the

A SHAHDARA 35S: IAA26-P108L-GFP
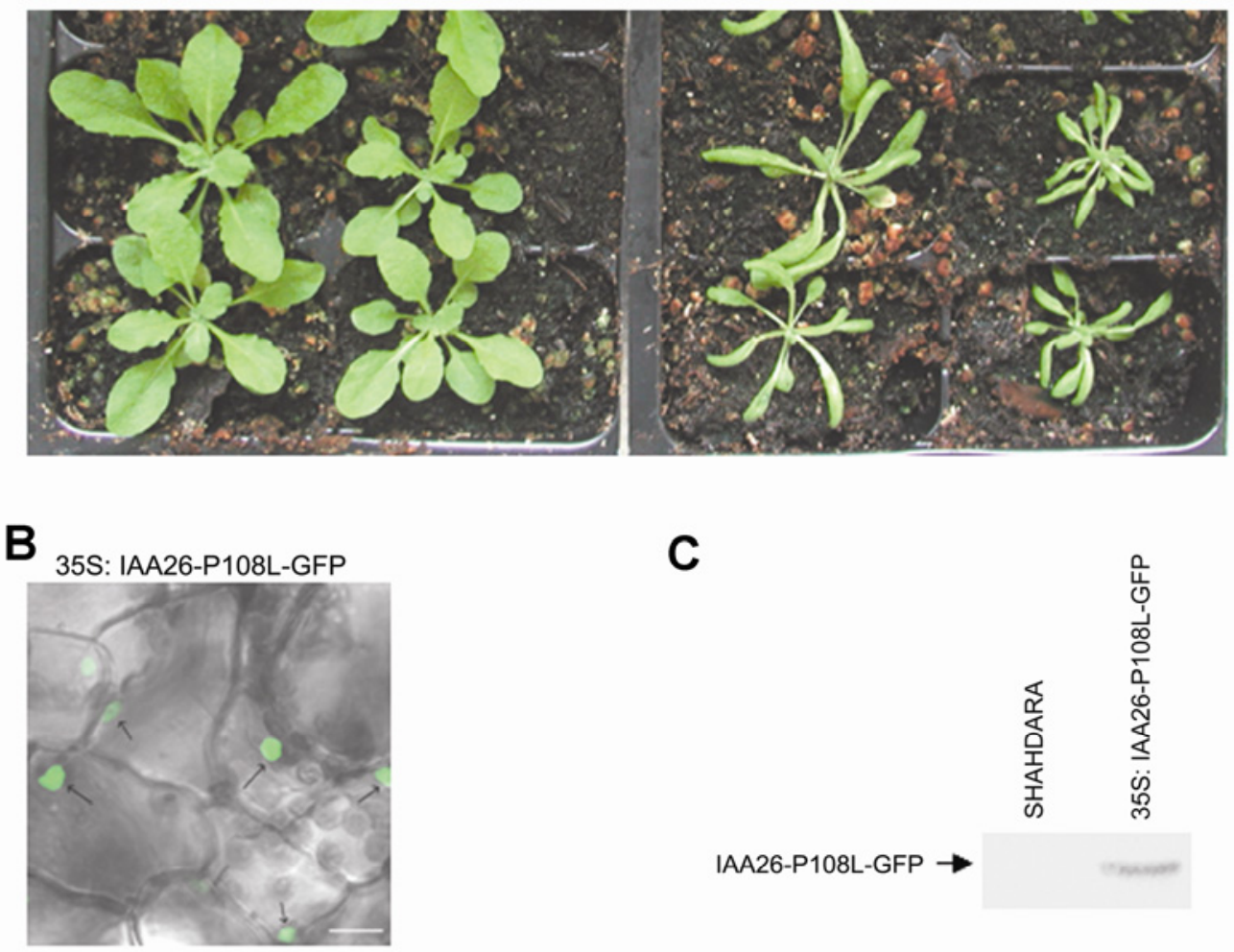

C
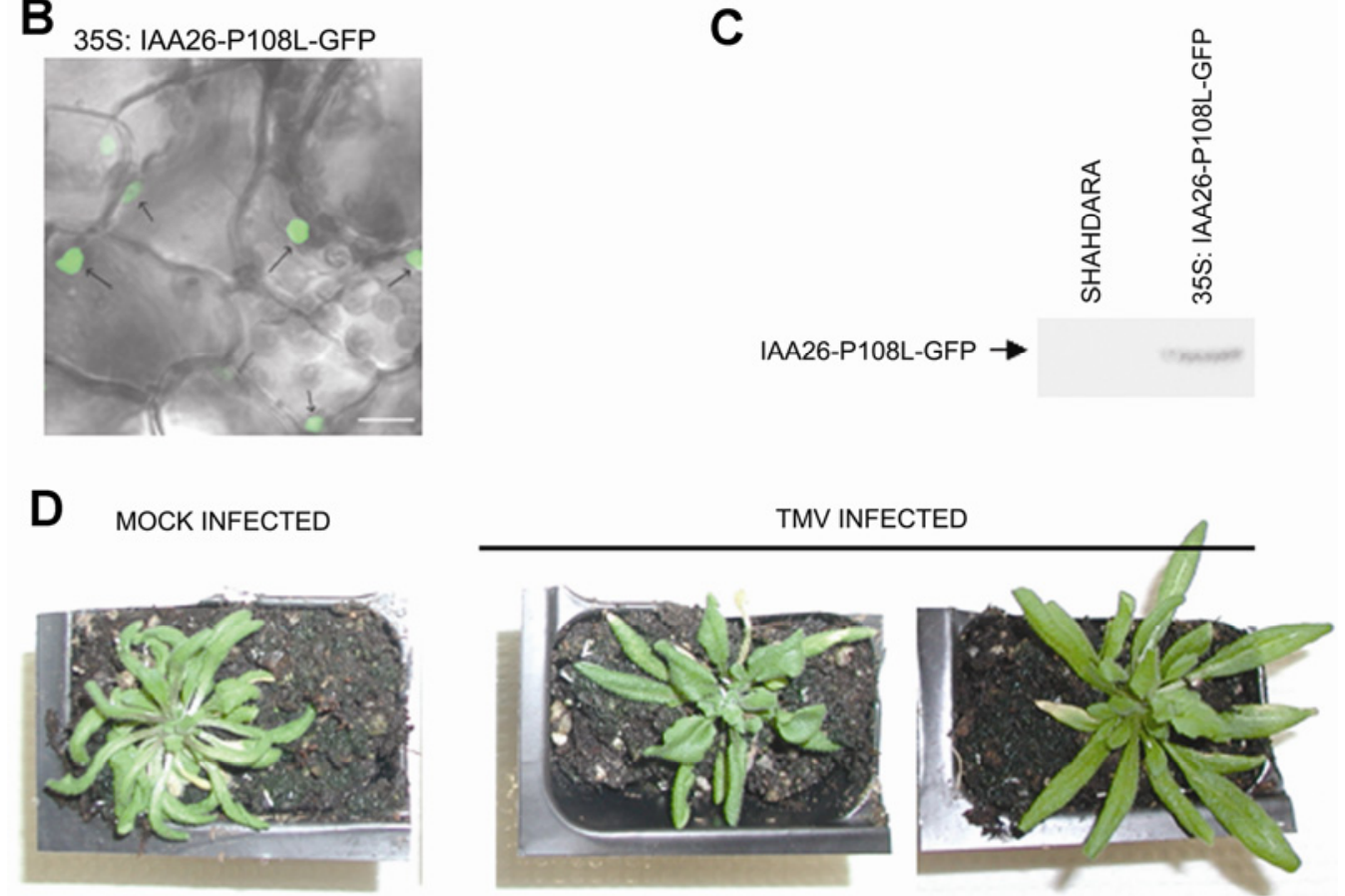

Fig. 7. Transgenic plants overexpressing indole acetic acid (IAA)26-P108L-green fluorescent protein (GFP) have an abnormal developmental phenotype that is attenuated during Tobacco mosaic virus (TMV) infection. A, Four-week old untransformed Arabidopsis thaliana ecotype Shahdara and 35S:IAA26P108H-GFP-transformed Shahdara plants. B, Confocal image of epidermal cells from a 2-week-old 35S:IAA26-P108H-GFP Shahdara leaf. Arrows indicate IAA26-P108H-GFP-derived nuclear florescence. C, Western immunoblot showing the detection of the IAA26-P108H-GFP fusion protein in transformed 5week-old leaf tissues. D, Transgenic 35S:IAA26-P108H-GFP plants treated with distilled water (Mock infected) or inoculated with TMV (TMV infected) and photographed 3 weeks postinoculation. 
conserved proline at position 108 in the IAA26-GFP construct was mutated to leucine, the resultant protein (IAA26-P108HGFP) was found to be stable even in the presence of high levels of auxin, and transgenic plants expressing the protein showed severe stunting, shortened petioles, and leaf curling, phenoltypes that are associated with altered auxin responses (Leyser et al. 1996; Liscum and Reed 2002). Although the point mutation appeared to stabilize IAA26, it did not compromise the interaction of IAA26 with TMV helicase. Based on this finding, we speculated that interaction with TMV replicase would reduce the accumulation of IAA26-P108H-GFP in the nucleus, thereby lessening the severity of the observed phenotype. Indeed, this was found to be the case with infected plants showing a reduction in the severity of the phenotype, with noticeable decreases in stunting and leaf curling. The change in phenotype was observed mainly in tissues that developed after inoculation and was consistent with the pattern of TMV spread in $A$. thaliana ecotype Shahdara (Dardick et al. 2000). The ability of a TMV infection to attenuate the abnormal developmental phenotype induced by the overexpression and accumulation of IAA26-P108H indicates that, even though the mutant protein is more stable, its interaction with replicase prevents it from reaching its appropriate site of action, thereby negating its dominant effect. Furthermore, the replicase levels produced during infection are sufficient to affect the function of interacting Aux/IAA proteins. This is further evidence that the interaction between the TMV replicase and Aux/IAA proteins can affect auxin-mediated pathways.

Virus disruption in plant hormone regulation previously has been attributed to the development of disease symptoms (Hull 2002; Jameson and Clarke 2002). Clearly, the effect of TMV replicase on Aux/IAA localization represents one direct mechanism through which this virus can influence hormone-controlled plant development. However, virus disruption of hormone regulation and symptom development is likely to be more complex, involving multiple virus and host components. For instance, studies on viral silencing suppressors have shown that viruses can indirectly affect the auxin response pathway by interfering with miRNAs involved in the regulation of certain auxin response factors (Chapman et al. 2004; Kasschau et al. 2003). In another study, interaction between the P2 protein of Rice dwarf virus and host ent-kaurene oxidases is correlated with the reduced biosynthesis of gibberellins and altered disease symptoms (Zhu et al. 2005). Additionally, although this is the first time that a TMV-encoded protein has been shown to alter the subcellular localization of a host protein, it appears that this mechanism for disrupting host function also is utilized by other viruses. Specifically, the coat protein of Turnip crinkle virus can effectively block the nuclear localization of an Arabidopsis transcription factor, TIP, involved in the induction of the hypersensitive response (Ren et al. 2005). Similarly, the P19 protein of Tomato bushy stunt virus interacts with and alters the nuclear localization of a family of plant ALY proteins thought to play a role in RNA transport (Uhrig et al. 2004). Thus, virus-directed alterations in host protein localization might represent a common mechanism that affects multiple virus-mediated host responses.

One remaining question is whether the virus gains anything from altering the localization of interacting Aux/IAA proteins. It is possible that disrupting the function of specific Aux/IAA proteins alters host metabolism to provide a more hospitable environment for virus replication and spread. For example, degradation of specific cellular proteins by herpes simplex virus1 results in more cells being committed to productive infections, particularly at low doses of input virus (Banks et al. 2003). However, partial loss of the TMV-IAA26 interaction by a helicase mutation did not affect overall levels of virus rep- lication and accumulation in whole leaf tissues, suggesting that this interaction does not significantly affect viral functions (Padmanabhan et al. 2005). The simplest explanation is that interactions with specific Aux/IAA proteins are inconsequential and independent of virus function. Though such interactions do not appear to be rate limiting for virus replication and spread, they can define the difference between a sensitive and tolerant host. Consistent with this possibility is the finding that many host systems can support virus replication and spread and yet display few if any phenotypic effects, indicating that the virus interferes little with normal host physiology (Hull 2002). The fact that TMV alters the localization and subsequent function of interacting Aux/IAA proteins suggests that this type of interaction plays a key role in defining host sensitivity.

\section{MATERIALS AND METHODS}

\section{Aux/IAA cloning and yeast two-hybrid analysis.}

Total RNA was extracted from A. thaliana Shahdara tissue using the RNeasy Plant Mini Kit (Qiagen, Valencia, CA, U.S.A.). cDNA synthesis was preformed using the SuperScript First-Strand Synthesis System for RT-PCR (Invitrogen, Carlsbad, CA, U.S.A.). Gene-specific primers containing the restrictions sites BamHI and XhoI were used to amplify all the Aux/IAAs except IAA27, for which BglII and Xho1 were used, and the IAA2 primers contained only the XhoI restriction sites. The primer sequences were GAGATCTCATGTCTGTAT CTGTAGCA and CTCGAGCTAGTTCCTGCTTCTGCA for IAA27, GGATCCGATGGAGGGTTATTCAAGA and CTCG AGTCATCTTCTCATTTTCTC for IAA18, CTCGAGGATGG CGTACGACGAGAAAGTC and CTCGAGTCATAAGGAAG AGTCTAG for IAA2, GGATCCAATGCGTGGTGTGTCAGA ATTGG and CTCGAG CTAAACAGGGTTGTTTCTTTG for IAA 12, GGATCCGATGGGAGGAGAAAAG and CTCGAG CTATTACTTGCCATGTTT for IAA28, GGATCCGATGGAA GGCGGTCCCGCTAGTGG and CTCGAGTCATAATATCAT CTGAGCTTTAC for IAA11, GGATCCGATGGAAAAAGTT GATGTTTATG and CTCGAGTTAAAGACCACCACAACC for IAA4, GGATCCGATTAATTTTGAGGC and CTCGAGTC AACTTCTGTTCTTGCAC for IAA 16, and GGATCCGATG AATGGTTTGCAAGAAG and CTCGAGCTACTTACCTACT CCAGCTCC for IAA10. The resulting PCR fragments were cloned into a pCRII-TOPO (Invitrogen) and subsequently ligated into appropriately digested pACT-GAL4 for yeast twohybrid analysis. All PCR derived clones were verified by sequencing.

Yeast two-hybrid interaction assays were preformed as previously described (Padmanabhan et al. 2005). Briefly, the L40 strain of Saccharomyces cerevisiae carrying the pLexA-TMV helicase bait was transformed with pACT-GAL4-Aux/IAA vectors. Quantitative $\beta$-galactosidase assays were performed on yeast cultures carrying both the pACT-bait and LexA-prey plasmids gown at $25^{\circ} \mathrm{C}$ in -Ura/-Trp/-Leu selection media as described by Miller (1972). Protein accumulation in representative pACT-GAL4 constructs encoding IAA27, IAA18, IAA12, and IAA2 ORFs were confirmed by Western immunoblot analysis using GAL4 specific antibodies.

\section{Transient expression constructs.}

The expression vector pCMC1100, containing a polylinker domain flanked by the Cauliflower mosaic virus CaMV 35S promoter and a polyadenylation signal, was used as the parental plasmid for all transient expression constructs (McCabe et al. 1988). The construction of pCMC-126-GFP, pCMC-126-781 GFP/pTMV-126_HEL-GFP, and pCMC-IAA26/PAP1-GFP were described previously (Figueira et al. 2002; Padmanabhan 
et al. 2005). PCR-based site-directed mutagenesis primers (GT GGTGGGTTGGCTTCCGGTTCGT and ACGAACCGGAAG CCAACCCACCAC) were used to create pCMC-IAA26P108H-GFP. DsRed constructs were prepared by replacing the GFP ORF in pCMC-IAA26-GFP with a PCR-modified DsRedII ORF (Clontech, Palo Alto, CA, U.S.A.) containing $5^{\prime}$ end $B s i \mathrm{WI}$ and $3^{\prime}$ end NciI sites. Full-length IAA2, IAA12, IAA18, and IAA27 cDNAs were obtained as described above and modified by PCR to contain $5^{\prime}$ end $K p n I$ and a $3^{\prime}$ end $B s i$ WI restriction sites. Individually modified Aux/IAA ORFs were ligated into similarly cut pCMC-GUS-GFP (Figueira et al. 2002), replacing the glucuronidase ORF. The GFP ORFs of individual pCMCAux/IAA-GFP constructs were replaced by DsRed as described above to create the pCMC-Aux/IAA-DsRed constructs.

\section{Transient expression assays.}

Transient expression assays were done as described previously (Figueira et al. 2002; Padmanabhan et al. 2005), using either onion epidermal peels or Nicotiana benthamiana leaf tissue. Briefly, a total of $4 \mu \mathrm{g}$ of plasmid DNA was ethanolprecipitated onto $0.5 \mathrm{mg}$ of tungsten particles $(1.3 \mu \mathrm{m}$ in diameter) (Bio-Rad, Hercules, CA, U.S.A.). DNA-coated particles were resuspended in $95 \%$ ethanol by sonication in a Brandon 2200 ultrasonic cleanser (Branson Equipment, Shelton, CT, U.S.A.) and loaded onto plastic filter screens (Gelman Sciences, Ann Arbor, MI, U.S.A.). Coated screens were dried and mounted into a particle inflow gun (Finer et al. 1992; Takeuchi et al. 1992) and a 50-ms pulse of helium (50 psi) was used to propel the particles into onion monolayers and leaf tissues mounted 2.5 and 3 in., respectively, below the filters. The tissues were incubated for 16 to $20 \mathrm{~h}$ at room temperature and mounted on glass slides in distilled water under coverslips. A Zeiss LSM510 laser scanning confocal microscope with $\times 10$ numerical aperture (NA) 0.8 dry and $\times 63$ NA 1.2 waterimmersion lenses (Carl Zeiss Inc., Thornwood, NY, U.S.A.) was used to visualize the samples. Excitation sources were 488 $\mathrm{nm}$ for GFP and $543 \mathrm{~nm}$ for DsRed. Images were modified using Zeiss LSM Imager Examiner software, version 3.0, and processed for printing with Adobe Photoshop (Grand Prairie, TX, U.S.A.).

\section{Auxin assays.}

pCMC-IAA26-GFP and pCMC-IAA26-P108H-GFP proteins were transiently expressed in onion epidermal cells as explained above. After a 14-h incubation, cells were mounted onto glass slides in distilled water under cover slips and fluorescent cells were examined by confocal microscopy for GFP expression. Approximately $500 \mu \mathrm{l}$ of $1 \mathrm{mM}$ IAA solution was added onto the surface of the onion monolayers and the cells re-visualized for GFP intensity and expression after $60 \mathrm{~min}$.

\section{Plant transformation and characterization.}

A derivative of the Agrobacterium binary transformation vector pBI121 (Clontech) was used to construct the IAA26 transformation vector. pCMC-IAA26-P108H-GFP was used as a template to PCR amplify the IAA26-P108H-GFP ORF using primers designed to introduce a $5^{\prime} \mathrm{XbaI}$ site (CTCTAGAATG GAAGGTTGTCCAAGAAAC) and a $3^{\prime}$ XhoI site (CTCGAGT TACTTGTACAGCTCGTCCATG). The resulting PCR product was digested with $X b a 1$ and $X h o I$ and inserted downstream of the CaMV $35 \mathrm{~S}$ promoter within a similarly digested pBI121 vector. The vector was introduced into Agrobacterium tumefaciens GV3101 for transformation into Arabidopsis thaliana ecotype Shahdara using the floral dip method (Clough and Bent 1998). Transformed seedlings were selected based on kanamycin resistance. Nine transgenic lines were obtained, of which five lines had a severe phenotype and four lines had an intermediate phenotype. T2 seed from line five, showing an intermediate phenotype, were used for phenotypic analysis and TMV inoculation. Protein extractions from Arabidopsis leaves were carried out as described by Osman and Buck (1996). Protein extracts were resolved on a $10 \%$ sodium dodecyl sulfide polyacrylamide gel and transferred to nitrocellulose paper (Fisher Scientific Company, Newark, DE, U.S.A.). Immunoblot analysis was performed using alkaline phosphataselabeled anti-GFP antibody (Clontech). TMV infections were confirmed by Western immunoblot analysis using replicasespecific antibodies.

\section{ACKNOWLEDGMENTS}

This work was supported in part by grants from the National Science Foundation (IBN-0113536) and the United States Department of Agriculture (0535319).

\section{LITERATURE CITED}

Abbink, T. E., de Vogel, J., Bol, J. F., and Linthorst, H. J. 2001. Induction of a hypersensitive response by chimeric helicase sequences of tobamoviruses $\mathrm{U} 1$ and $\mathrm{Ob}$ in N-carrying tobacco. Mol. Plant-Microbe Interact. 14:1086-1095.

Abbink, T. E., Peart, J. R., Mos, T. N., Baulcombe, D. C., Bol, J. F., and Linthorst, H .J. 2002. Silencing of a gene encoding a protein component of the oxygen-evolving complex of photosystem II enhances virus replication in plants. Virology 295:307-319.

Abel, S., Oeller, P. W., and Theologis, A. 1994. Early auxin-induced genes encode short-lived nuclear proteins. Proc. Natl. Acad. Sci. U.S.A. 91:326-330.

Asurmendi, S., Berg, R. H., Koo, J. C., and Beachy, R. N. 2004. Coat protein regulates formation of replication complexes during tobacco mosaic virus infection. Proc. Natl. Acad. Sci. U.S.A. 101:1415-1420.

Banks, L., Pim, D., and Thomas, M. 2003. Viruses and 26S proteasome: hacking into destruction. Trends Biochem. Sci. 28:452-459.

Bao, Y., Carter, S. A., and Nelson, R. S. 1996. The 126- and 183-kilodalton proteins of tobacco mosaic virus, and not their common nucleotide sequence, control mosaic symptom formation in tobacco. J. Virol. 70:6378-6383.

Beachy, R. N., and Zaitlin, M. 1975. Replication of tobacco mosaic virus, VI Replicative intermediate and TMV-RNA-related RNAs associated with polyribosomes. Virology 63:84-97.

Bilgin, D. D., Liu, Y., Schiff, M., and Dinesh-Kumar, S.P. 2003. P58(IPK), a plant ortholog of double-stranded RNA-dependent protein kinase PKR inhibitor, functions in viral pathogenesis. Dev. Cell 4:651-661.

Chapman, E. J., Prokhnevsky, A.I., Gopinath, K., Dolja, V. V., and Carrington, J. C. 2004. Viral RNA silencing suppressors inhibit the microRNA pathway at an intermediate step. Genes Dev. 18:1179-1186.

Clough, S. J., and Bent, A. 1998. Floral dip: a simplified method for Agrobacterium-mediated transformation of Arabidopsis thaliana. Plant J. 16:735-743.

Dardick, C. D., Golem, S., and Culver, J. N. 2000. Susceptibility and symptom development in Arabidopsis thaliana to Tobacco mosaic virus is influenced by virus cell to cell movement. Mol. Plant-Microbe Interact. 13:1139-1144.

Dharmasiri, N., and Estelle. M. 2004. Auxin signaling and regulated protein degradation. Trends Plant Sci. 9:302-308.

Ding, X. S., Liu, J., Cheng, N. H., Folimonov, A., Hou, Y. M., Bao, Y., Katagi, C., Carter, S. A., and Nelson, R. S. 2004. The Tobacco mosaic virus $126-\mathrm{kDa}$ protein associated with virus replication and movement suppresses RNA silencing. Mol. Plant-Microbe Interact.17:583-592.

Dunigan, D. D., and Zaitlin, M. 1990. Capping of tobacco mosaic virus RNA. Analysis of viral-coded guanylyltransferase-like activity. J. Biol. Chem. 265:7779-7786.

Erickson, F. L., Holzberg, S., Calderon-Urrea, A., Handley, V., Axtell, M., Corr, C., and Baker, B. 1999. The helicase domain of the TMV replicase proteins induces the $\mathrm{N}$-mediated defense response in tobacco. Plant J. 18:67-75.

Figueira, A., Golem, S., Goregaoker, S. P., and Culver, J. N. 2002. A nuclear localization signal and a membrane association domain contribute to the cellular localization of the Tobacco mosaic virus 126-kDa replicase protein. Virology 301:81-89.

Finer, J. J., Vain, P., Jones, M. W., and McMullin, M. D. 1992. Development of the particle inflow gun for DNA delivery to plant cells. Plant Cell Rep. 11:323-328. 
Goregaoker, S. P., and Culver, J. N. 2003. Oligomerization and activity of the helicase domain of the Tobacco mosaic virus 126- and 183-kilodalton replicase proteins. J. Virol. 77:3549-3556.

Goregaoker, S. P., Lewandowski, D., and Culver, J. N. 2001. Identification and functional analysis of an interaction between domains of the 126/183-kDa replicase-associated proteins of Tobacco mosaic virus. Virology 282:320-328.

Gray, W. M., Kepinski, S., Rouse, D., Leyser, O., and Estelle, M. 2001. Auxin regulates SCF(TIR1)-dependent degradation of AUX/IAA proteins. Nature 414:271-276.

Heinlein M., Padgett, H. S., Gens, J. S., Pickard, B. G., Casper, S. J., Epel, B .L., and Beachy, R. N. 1998. Changing patterns of localization of the tobacco mosaic virus movement protein and replicase to the endoplasmic reticulum and microtubules during infection. Plant Cell 10:1107-1120.

Hirashima, K., and Watanabe, Y. 2001. Tobamovirus replicase coding region is involved in cell-to-cell movement. J Virol. 75:8831-8836.

Hull, R. 2002. Matthews' Plant Virology. Academic Press, London.

Jameson, P. E., and Clarke, S. F. 2002. Hormone-virus interactions in plants. CRC Crit. Rev. Plant Sci. 21:205-228.

Kasschau, K. D., Xie, Z., Allen, E., Llave, C., Chapman, E. J., Krizan, K. A., and Carrington, J. C. 2003. P1/HC-Pro, a viral suppressor of RNA silencing, interferes with Arabidopsis development and miRNA function. Dev Cell. 4:205-217.

Kepinski, S., and Leyser, O. 2002. Ubiquitination and auxin signaling: a degrading story. Plant Cell 14:S81-S95.

Kepinski, S., and Leyser, O. 2004. Auxin-induced SCFTIR1-Aux/IAA interaction involves stable modification of the SCFTIR1 complex. Proc. Natl. Acad. Sci. U.S.A. 101:12381-12386.

Kepinski, S., and Leyser, O. 2005. The Arabidopsis F-box protein TIR1 is an auxin receptor. Nature 435:446-451.

Kim, J., Harter, K. and Theologis, A. 1997. Protein-protein interactions among the Aux/IAA proteins. Proc. Natl. Acad. Sci. U.S.A. 94:1178611791.

Kubota, K., Tsuda, S., Tamai, A., and Meshi, T. 2003. Tomato mosaic virus replication protein suppresses virus-targeted posttranscriptional gene silencing. J. Virol. 77:11016-11026.

Leyser, H. M. O., Pickett, F. B., Dharmasiri, S. and Estelle, M. 1996. Mutations in the AXR3 gene of Arabidopsis result in altered auxin response including ectopic expression from the SAUR-AC1 promoter. Plant J. 10:403-413.

Leyser, O. 2002. Molecular genetics of Auxin signaling. Annu. Rev. Plant Biol. 53:377-398.

Liscum, E., and Reed, J. W. 2002. Genetics of Aux/IAA and ARF action in plant growth and development. Plant Mol. Biol. 49:387-400.

Liu, J. Z., Blancaflor, E. B., and Nelson, R. S. 2005. The Tobacco mosaic virus 126-kilodalton protein, a constituent of the virus replication complex, alone or within the complex aligns with and traffics along microfilaments. Plant Physiol. 138:1853-1865.

Más, P., and Beachy, R. N. 1999. Replication of Tobacco mosaic virus on endoplasmic reticulum and role of the cytoskeleton and virus movement protein in intercellular distribution of viral RNA. J. Cell Biol. 147:945958

Más, P., and Beachy, R. N. 2000. Role of microtubules in the intracellular distribution of Tobacco mosaic virus movement protein. Proc. Natl. Acad. Sci. U.S.A. 97:12345-12349.

Mathews, R. E. F. 1981. Plant Virology. Academic Press, London.

McCabe, D. E., Swain, W. F., Martinell, B. J., and Christou, P. 1988. Stable transformation of soybean (Glycine max) by particle bombardment. Biotechnology 6:923-926.

Merits, A., Kettunen, R., Mäkinen, K., Lampio, A., Auvinen, P., Kääriäinen, L., and Ahola , T. 1999. Virus-specific capping of tobacco mosaic virus RNA: methylation of GTP prior to formation of covalent complex p126$\mathrm{m}^{7}$ GMP. FEBS (Fed. Eur. Biochem. Soc.) Lett. 16:45-48.

Miller, J. H. 1972. Experiments in Molecular Genetics. Cold Spring Harbor Laboratory Press, Cold Spring Harbor, NY, U.S.A.

Nagpal, P., Walker, L .M., Young, J. C., Sonawala, A., Timte, C., Estelle, M., and Reed, J.W. 2000. AXR2 encodes a member of the Aux/IAA protein family. Plant Physiol. 123:563-574.

Osman. T. A., and Buck, K. W. 1996. Complete replication in vitro of Tobacco mosaic virus RNA by a template-dependent, membrane-bound RNA polymerase. J. Virol. 70:6227-6234.

Osman, T. A., and Buck, K. W. 1997. The Tobacco mosaic virus RNA polymerase complex contains a plant protein related to the RNA-binding subunit of yeast eIF-3. J. Virol. 71:6075-6082.
Osman, T. A., and Buck, K. W. 2003. Identification of a region of the Tobacco mosaic virus 126- and 183-kilodalton replication proteins which binds specifically to the viral 3 '-terminal tRNA-like structure. J. Virol. 77:8669-8675

Ouellet, F., Overvoorde, P. J., and Theologis, A. 2001. IAA17/AXR3. Biochemical insight into an auxin mutant phenotype. Plant Cell 13:829841.

Padgett, H. S., and Beachy, R. N. 1993. Analysis of a tobacco mosaic virus strain capable of overcoming $\mathrm{N}$ gene-mediated resistance. Plant Cell 5:577-586.

Padmanabhan, M. S., Goregaoker, S. P., Golem, S., Shiferaw, H., and Culver, J. N. 2005. Interaction of the Tobacco mosaic virus replicase protein with the Aux/IAA protein PAP1/IAA26 is associated with disease development. J. Virol. 79:2549-2558.

Park, J. Y., Kim, H. J., and Kim, J. 2002. Mutation in domain II of IAA1 confers diverse auxin-related phenotypes and represses auxin-activated expression of Aux/IAA genes in steroid regulator-inducible system. Plant J. 32:669-683.

Ramos, J. A., Zenser, N., Leyser, O., and Callis, J. 2001. Rapid degradation of auxin/indole acetic acid proteins requires conserved amino acids of domain II and is proteasome dependent. Plant Cell 13:2349-2360.

Reed, J. W. 2001. Roles and activities of Aux/IAA proteins in Arabidopsis. Trends Plant Sci. 6:420-425.

Reichel, C., and Beachy, R. N. 1998. Tobacco mosaic virus infection induces severe morphological changes of the endoplasmic reticulum. Proc. Natl. Acad. Sci. U.S.A. 15:11169-11174.

Ren, T., Qu, F., and Morris, T. J. 2005. The nuclear localization of the Arabidopsis transcription factor TIP is blocked by its interaction with the coat protein of Turnip crinkle virus. Virology 331:316-324.

Rogg, L. E., Lasswell, J., and Bartel, B. 2001. A gain-of-function mutation in IAA28 suppresses lateral root development. Plant Cell 13:465-480.

Rouse, D., Mackay, P., Stirnberg, P., Estelle, M., and Leyser, O. 1998 Changes in auxin response from mutations in an AUX/IAA gene. Science 279:1371-1373.

Shalla, T. A. 1964. Assembly and aggregation of tobacco mosaic virus in tomato leaflets. J. Cell Biol. 21:253-264.

Shintaku, M. H., Carter, S. A., Bao, Y., and Nelson, R. S. 1996. Mapping nucleotides in the $126-\mathrm{kDa}$ protein gene that control the differential symptoms induced by two strains of tobacco mosaic virus. Virology 221:218-225

Szécsi, J., Ding, X. S., Lim, C. O., Bendahmane, M., Cho, M. J., Nelson, R. S., and Beachy, R. N. 1999. Development of Tobacco mosaic virus infection sites in Nicotiana benthamiana. Mol. Plant-Microbe Interact. 12:143-152.

Takeuchi, Y., Dotson, M., and Keen, N. T. 1992. Plant transformation: simple particle bombardment device based on flowing helium. Plant Mol. Biol. 18:835-839.

Tian, Q., Nagpal, P., and Reed, J. W. 2003. Regulation of Arabidopsis SHY2/IAA3 protein turnover. Plant J. 36:643-651.

Uhrig, J. F., Canto, T., Marshall, D., and MacFarlane, S. A.. 2004. Relocalization of nuclear ALY proteins to the cytoplasm by the tomato bushy stunt virus P19 pathogenicity protein. Plant Physiol. 135:24112423

Ulmasov, T., Hagen, G., and Guilfoyle, T. J. 1997a. ARF1, a transcription factor that binds to auxin response elements. Science 276:1865-1868.

Ulmasov, T., Liu, Z. B., Hagen, G., and Guilfoyle, T. J. 1995. Composite structure of auxin response elements. Plant Cell 7:1611-1623.

Ulmasov, T., Murfett, J., Hagen, G., and Guilfoyle, T. J. 1997b. Aux/IAA proteins repress expression of reporters genes containing natural and highly active synthetic auxin response elements. Plant Cell 9:19631971

Yamanaka, T., Ohta, T., Takahashi, M., Meshi, T., Schmidt, R., Dean, C., Naito, S., and Ishikawa, M. 2000. TOM1, an Arabidopsis gene required for efficient multiplication of a Tobamovirus, encodes a putative transmembrane protein. Proc. Natl. Acad. Sci. U.S.A. 97:10107-10112.

Zenser, N., Dreher, K. A., Edwards, S. R., and Callis, J. 2003.Acceleration of Aux/IAA proteolysis is specific for auxin and independent of AXR1. Plant J. 35:285-294.

Zhou, X., Carranco, R., Vitha, S., and Hall T. C. 2005. The dark side of green fluorescent protein. New Phytol. 168:313-322.

Zhu, S., Gao, F., Cao, X., Chen, M., Ye, G., Wei, C., and Li, Y. 2005. The rice dwarf virus $\mathrm{P} 2$ protein interacts with ent-kaurene oxidases in vivo, leading to reduced biosynthesis of gibberellins and rice dwarf symptoms. Plant Physiol. 139:1935-1945.

An error in the description of the proteolysis-resistant IAA26 protein was published in the original article. The mutation introduced in the IAA26 open reading frame led to an amino acid substitution of Proline to Histidine at position 108 (IAA26-P108H) and not Proline to Leucine (IAA26$\mathrm{P} 108 \mathrm{~L}$ ) as reported in the paper. The P108H mutation occurs in all the proteolysis-resistant IAA26 constructs used in this study. Thus, this change does not alter the original findings of the paper. The online text was changed on May 20, 2015. 